\title{
Sudden Unexpected Death of a Term Fetus in an Anticardiolipin-Positive Mother
}

\author{
Giulia Ottaviani, M.D., ${ }^{1}$ Anna M. Lavezzi, M.D., ${ }^{1}$ Lino Rossi, M.D., ${ }^{1}$ \\ and Luigi Matturri, M.D., Ph.D.'
}

A term male fetus suddenly and unexpectedly died in utero at the 40th week of gestation. The mother had a regular and unremarkable pregnancy except for an anticardiolipin antibodies positivity. The histological examination of the cardiac conduction system showed islands of conduction tissue in the central fibrous body, known as persistent fetal dispersion. The brainstem examination revealed a severe bilateral hypoplasia of the arcuate nucleus. This morphological finding has been described in more than $35 \%$ of our stillborn as well as sudden infant death syndrome (SIDS) cases, independently from the presence of anticardiolipin antibodies. Some authors have given emphasis to the possible lethal association of maternal autoantibodies and QT prolongation. Our findings emphasize the need of an accurate postmortem examination including the study of brainstem and cardiac conduction system in every case of unexpected late intrauterine death, following the same standardized autopsy protocol adopted in SIDS cases.

KEYWORDS: Stillbirth, sudden unexplained intrauterine death, anticardiolipin antibodies

Stillbirth is defined as late intrauterine death before the complete expulsion of the fetus from the mother. ${ }^{1}$ A high frequency of hypoplasia of the arcuate nucleus occurs in fetuses who have died sine causa in a similar manner to that observed in sudden infant death syndrome (SIDS). ${ }^{2}$

Herein we report a case of a term fetus dying suddenly and unexpectedly, delivered by cesarean section to a 35-year-old mother presenting with elevated anticardiolipin antibodies.

For several years we have been interested in the histological study of bulbar cardiorespiratory centers in victims of SIDS and sudden intrauterine unexplained death (SIUD)., ${ }^{2,3}$ The importance and frequency of sudden death in late fetuses and infants prompted us to analyze in more detail the brainstem

American Journal of Perinatology, Volume 21, Number 2, 2004. Address for correspondence and reprint requests: Luigi Matturri, M.D., Ph.D., Institute of Pathology, University of Milan, Via della Commenda, 19, 20122 Milan, Italy. ${ }^{1}$ Institute of Pathology, University of Milan, Italy. Copyright (C) 2004 by Thieme Medical Publishers, Inc., 333 Seventh Avenue, New York, NY 10001, USA. Tel: +1(212) 584-4662. 07351631,p;2004,21,02,079,084,ftx,en;ajp39370x. 
changes in these infants. Preliminary findings have been reported in abstract form. ${ }^{4}$

\section{CASE REPORT}

A term male fetus suddenly and unexpectedly died in utero at the 40th week of gestation. He was delivered by cesarean section. The mother, a 35year-old woman, gravida 2 , para $1+1$, had an unremarkable pregnancy except for a mild positivity for anticardiolipin antibodies. Antenatal screening was negative for lupus anticoagulant. During the pregnancy she had been treated with low-dose aspirin.

There was no significant family history relevant to the case. An antenatal electrocardiogram (ECG) recording of the fetus was reported to be normal.

Postmortem examination was requested with clinically suspected atrioventricular block caused by anticardiolipin antibodies.

\section{MATERIALS AND METHODS}

A complete autopsy was performed, according to the autopsy protocol usually followed at our institute in cases of sudden infant death, particularly including the assessment of the cardiac conduction system and the central and peripheral autonomic nervous systems. ${ }^{2,3,5,6}$ Multiple samples of all organs were fixed in 10\% buffered formalin, processed, and embedded in paraffin. Five-micrometer sections were stained with hematoxylin and eosin. The heart was examined for pathologic changes in the atria, septum, ventricles, pericardium, endocardium, and coronary arteries. Sections of the myocardium were stained with hematoxylin and eosin and Heidenhain's trichrome (azan). Histological observations were focused on the cardiac conduction system, which was removed in two blocks: the first included the sinoatrial node and the crista terminalis; the second contained the atrioventricular node, His bundle, bifurcation, and bundle branches.

Transverse serial $5-\mu \mathrm{m}$ sections were made through the entire pons and medulla oblongata, and were stained with hematoxylin and eosin, Bielschowsky, and Klüver-Barrera stains. The pertinent nuclei were outlined, namely the parabrachial/Kölliker-Fuse complex in the pons, the arcuate nucleus, the hypoglossus nucleus, the dorsal vagus motor nucleus, the tract solitarius nucleus, the ambiguous nucleus, the trigeminal tractus and nucleus, and the ventrolateral reticular formation in the medulla oblongata. Plates in the atlas of Olszewski and Baxter were used for reference. ${ }^{7} \mathrm{~A}$ morphometric analysis of the brainstem was performed with an Image-Pro Plus Image analyzer (Media Cybernetics, Silver Spring, MD). The volume of the arcuate nucleus was measured by 3-dimensional reconstruction.

The lung examination to determine the stage of development was evaluated on the basis of a macroscopic criterion, namely the correlation between lungs weight and body weight and according to microscopic criteria; that is, the presence of cartilaginous bronchi up to the distal peripheral level and the radial alveolar count. ${ }^{8}$

\section{PATHOLOGIC FINDINGS}

At autopsy, the fetus was described as a welldeveloped, well-nourished white infant, with body length of $35 \mathrm{~cm}$ and body weight of $3180 \mathrm{~g}$. The external and internal examinations were entirely normal for gestational age and sex.

The sinoatrial node, atrioventricular node, His bundle, and bundle branches were found to be normal histologically. Close to the atrioventricular node, islands of conduction tissue in the central fibrous body, known as fetal dispersion of the atrioventricular node, ${ }^{5,9}$ were observed (Fig. 1).

The histological examination of the brainstem revealed a bilateral hypoplasia of the arcuate 


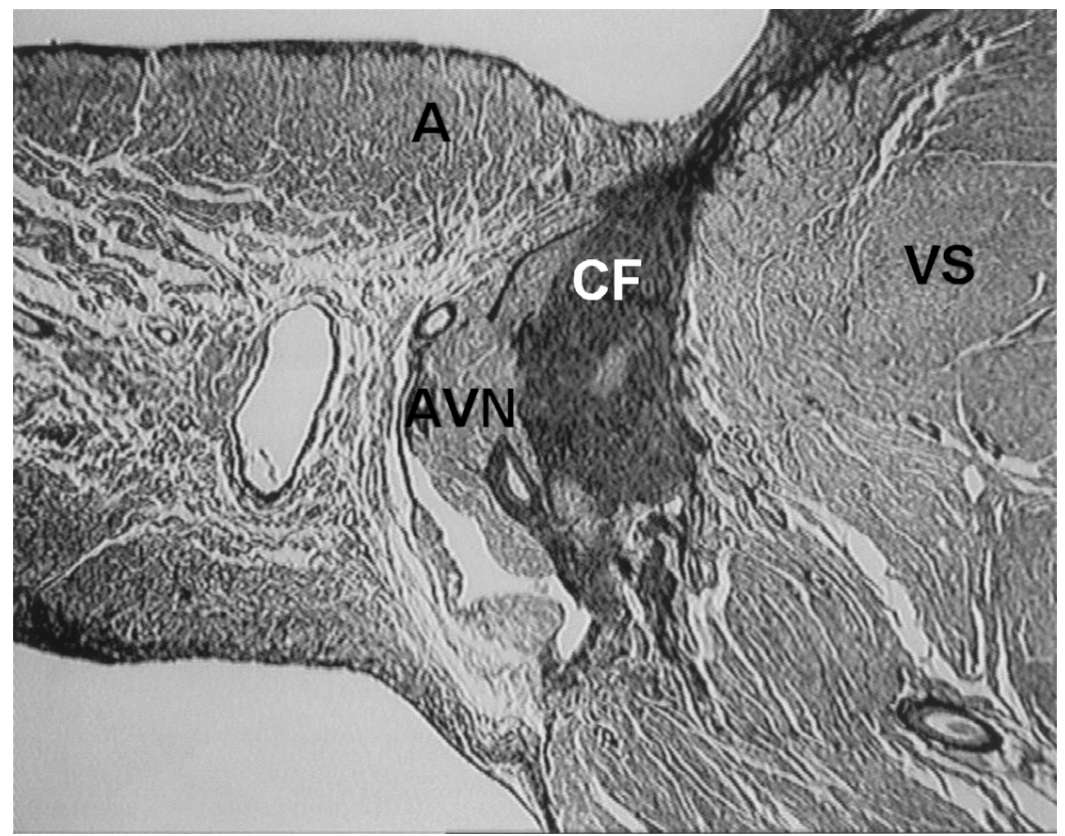

Figure 1 Islands of conduction tissue embedded in the central fibrous body. The atrioventricular node is well developed and centered around the homonymous artery. CF, central fibrous body; AVN, atrioventricular node; VS, interventricular septum; A, atrium. Trichromic Heidenhain stain, 25x.

nucleus (Fig. 2) and hypoplasia of the reticular formation. No pulmonary hypoplasia was observed.

\section{DIscussion}

Anticardiolipin antibodies in pregnancy are associated with recurrent fetal death. Several treatments have been proposed: low-dose aspirin, corticosteroids, heparin, and intravenous immunoglobulin. ${ }^{10}$

Pre- or perinatal death from heart block as a result of severe autoimmune lesions of the atrioventricular junction has been reported. ${ }^{11,12}$ In this case, the atrioventricular node and the entire cardiac conduction system appeared to be well developed. Our findings rule out the clinically suspected atrioventricular block caused by the anticardiolipin antibodies. The islands of conducting tissue detected in the central fibrous body (Fig. 1) represent the pattern of fetal dispersion and are considered to be a physiological process during fetal life.
The histological examination of the brainstem on serial sections revealed a severe bilateral hypoplasia of the arcuate nucleus (Fig. 2), which is an important chemoreceptor center for the control of breathing activity, located on the medullary ventral surface. This morphological finding has been described in more than $35 \%$ of our stillborn as well as SIDS cases. ${ }^{2,3,13}$

The hypodevelopment of the arcuate nucleus, which is often associated with respiratory reticular formation hypoplasia and pulmonary hypoplasia in stillborn fetuses, ${ }^{13}$ is analogous in SIUD and SIDS cases, ${ }^{2,3}$ independent of the presence of anticardiolipin antibodies.

Recently, some authors ${ }^{11,12}$ have emphasized the possible lethal association of maternal autoantibodies and QT prolongation, but they did not perform a postmortem examination of the brainstem, which is considered to be of great importance by the present authors. Furthermore, in this case, the antenatal ECG did not show a prolongation of the QT interval. 


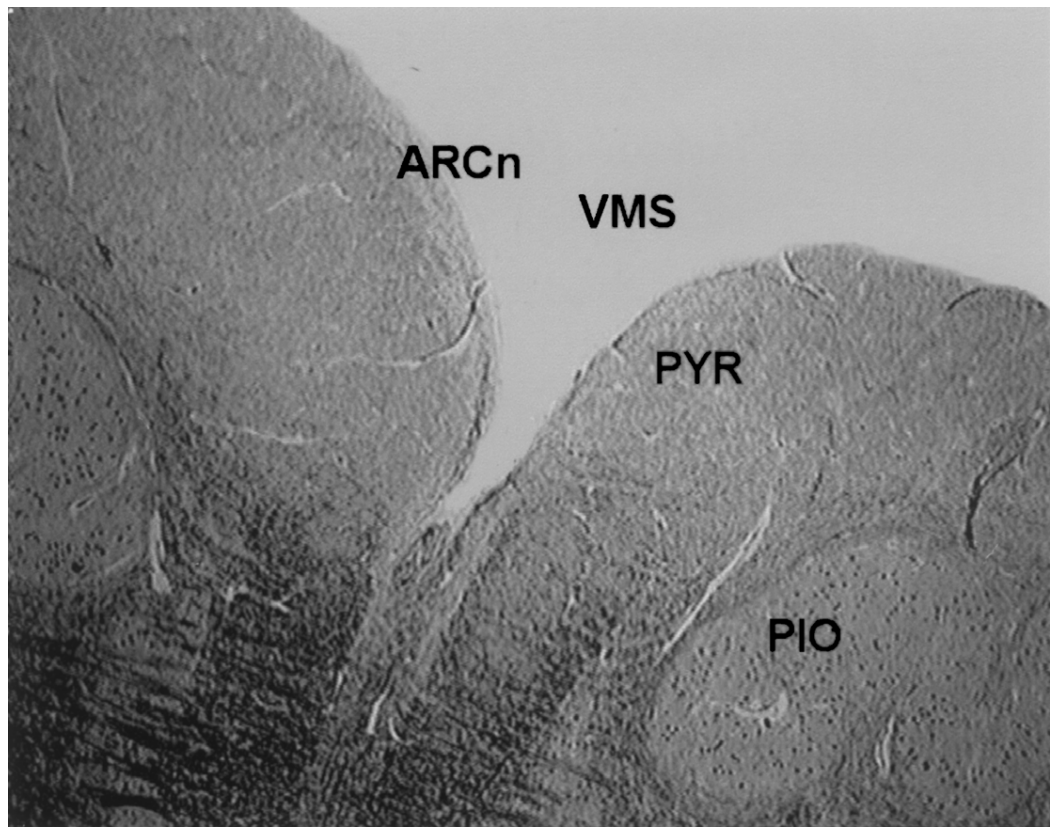

Figure 2 Bilateral hypoplasia of the arcuate nucleus (ARCn). VMS, ventral medullary surface; PIO, principal inferior olive; PYR, pyramid. Klüver-Barrera stain, 25×.

Our findings emphasize the need of an accurate postmortem examination including the study of the brainstem and cardiac conduction system on serial sections in every case of SIUD, following a standardized autopsy protocol similar to the one adopted in SIDS cases.

\section{ACKNOWLEDGMENTS}

The authors wish to express their gratitude to Mrs. Graziella Alfonsi for skillful technical cooperation. Appreciation is extended to Mrs. Delfina Tosi for her advice and assistance with the photographic prints. This work was supported by the Lombardy Region project 49210-243.00: Program of Research and Intervention for the Reduction of the Risk of Sudden Infant Death and Unexpected Fetal Death.

\section{REFERENCES}

1. Kalousek DK, Gilbert-Barness E. Causes of stillbirth and neonatal death. In: Gilbert-Barness E, ed. Potter's
Pathology of the Fetus and Infant. St. Louis, MO: Mosby; 1997:128-162

2. Matturri L, Minoli I, Lavezzi AM, Cappellini A, Ramos S, Rossi L. Hypoplasia of medullary arcuate nucleus in unexpected late fetal death (stillborn infants): a pathologic study. Pediatrics 2002;109:E43

3. Matturri L, Biondo B, Suàrez-Mier MP, Rossi L. Brain stem lesions in the sudden infant death syndrome: variability in the hypoplasia of the arcuate nucleus. Acta Neuropathol (Berl) 2002;104:12-20

4. Rossi L, Matturri L, Ottaviani G. Sudden unexpected death of a term fetus with maternal anti-cardiolipin antibodies. In: Proceedings of the 7th SIDS International Conference, Florence, Italy; August 31-September 4, 2002: 137

5. Matturri L, Ottaviani G, Ramos SG, Rossi L. Sudden infant death syndrome (SIDS): a study of cardiac conduction system. Cardiovasc Pathol 2000;9:137145

6. Rossi L. Salvage the pacemaker at autopsy. Am Heart J 1978;95:540-541

7. Olsezewski J, Baxter D. Cytoarchitecture of the Human Brain Stem. Basel, Switzerland: Karger; 1982

8. Askenazi SS, Perlman M. Pulmonary hypoplasia: lung weight and radial alveolar count as criteria of diagnosis. Arch Dis Child 1979;54:614-618

9. James TN, Marshall TK. De subitaneis mortibus XVIII. Persistent fetal dispersion of the atrio-ventricular node and His bundle within the central fibrous body. Circulation 1976;53:1026-1034 
10. Vinatier D, Dufour P, Cosson M, Houpeau JL. Antiphospholipid syndrome and recurrent miscarriages. Eur J Obstet Gynecol Reprod Biol 2001;96:37-50

11. Cimaz R, Stramba-Badiale M, Brucato A, Catelli L, Panzeri P, Meroni PL. QT interval prolongation in asymptomatic anti-SSA/Ro-positive infants without congenital heart block. Arthritis Rheum 2000;43:10491053
12. Brucato A, Frassi M, Franceschini F, et al. Risk of congenital heart block in newborns of mothers anti-Ro/ SSA antibodies detected by counterimmunoelectrophoresis: a prospective study of 100 women. Arthritis Rheum 2001;44:1832-1835

13. Matturri L, Lavezzi AM, Minoli I, et al. Association between pulmonary hypoplasia and hypoplasia of arcuate nucleus in stillbirth. J Perinatol 2003;23:328-332 